\title{
Contemporary free-form pavilions with the Delaunay gridshell patterns
}

\author{
Anna Stefańska, ${ }^{1, *}$, and Ewelina Gawell ${ }^{1}$ \\ ${ }^{1}$ Department of Structure Design, Construction and Technical Infrastructure, Faculty of Architecture, Warsaw University of Technology, \\ Poland
}

\begin{abstract}
The design of unique gridshell forms has become a key factor in effective interdisciplinary designing solutions, both architecturally aesthetic and structurally efficient. Modern bionic tendencies allow designers to implement organic shapes through proportion and mimicking the biological, developmental process by understanding the logic of the structural forms occurring in Nature. The improvement of digital tools based on algorithmic codes has enabled architects to implement their bold designs based on Nature's technologies' logic. The purpose of the research was to identify how the mathematical algorithms found in Nature collaborate with parametric designing influence structural optimization of the free-form structures. The Delaunay divisions used in the gridshells form-finding were obtained through various generative modeling algorithms. The case studies demonstrate how mathematical algorithms, such as structural dynamic relaxation, can support architectural aesthetic and structural optimization processes. The paper concludes that proper generative algorithmic design can compromise design diversity and search for efficiency.
\end{abstract}

\section{Introduction}

A characteristic feature of modern architectural design is increasingly complex structural forms. The original, bionic shapes of the 21 st-century buildings are created due to the creative search and the changing philosophy in architectural design. Modern bionic tendencies support the search for synergistic solutions that combine the form's aesthetics with structural logic [1]. The analogies between architectural design and morphogenesis of biological forms have increased the interest in bionic structures. Computational calculation methods lead to improved modeling tools and the fabrication of building elements and even whole objects [2]. Currently, architects can design spatial forms with very complex structures. Algorithmization of the supporting design tools provides new opportunities, also creates new challenges, including rationalization, which necessitates a greater focus on conceptual modeling in the design process. The article analyzes pavilions as free-form canopies that are small scale architectural objects with a relatively uncomplicated function. The design of structural forms with computational tools and already the known mathematical models justify contemporary free-form structures. The research has focused on applying bionic formulas such as the Delaunay pattern and catenary model geometries for design exploration at the conceptual design stage. One of the most interesting bionic methods of discretization of structural surfaces is Delaunay triangulation, where examples can be found in the patterns of a dragonfly wing, giraffe's mottled skin, or a turtle's shell. Furthermore, catenary models can be considered to be the most efficient geometries basing on Nature's observation.

\section{Literature review}

The pursuit of the implementation of Nature's technologies in architecture takes place on several levels - the most common and intuitive is the imitation of organic forms. The goal may be to search for innovative tectonic solutions, not necessarily resulting from structural logic.

The algorithmization of modern modeling tools based on the established parameters provided architects with new possibilities, among others, to generate fractal structures. It became an impulse to create bionic structures in contemporary architecture to imitate Nature's technology through the shape, structure, and even the development processes [3] [4]. An intriguing example of this is presented in the "Virtual plants" installation designed by UVA (United Visual Artist) in 2009 for an exhibition organized by the Natural History Museum in London on the occasion of Darwin's 200th birthday. The authors of the installation designed an algorithm responsible for the virtual plant growth process in a virtual environment. The project aroused great interest among architects who noticed clear analogies between the 'plants-models' competing with each other for, among others, access to the virtual sun and real-world buildings that are faced with similar

\footnotetext{
* Corresponding author: anna.stefanska2@pw.edu.pl
} 
problems. Thanks to generative modeling tools, the fascination with bionics in architecture has gained a dynamic pace, and the search for spatial solutions outside the Euclidean geometry became common [5].

Optimization is an essential element in the design of modern technical architectural structures. In the context of creative designs and exploration, form-finding and topology deserve special attention at the intersection of architecture and structure. Confucius's original sentence, „, The hardest thing of all is to find a black cat in a dark room, especially if there is no cat in it," was paraphrased by the mathematician Andre Cherkaev. There is no golden ratio, and the skillful use of possible variants is most important - this is optimization, according to A. Cherkaev. Optimization was compared to: "searching for a black cat in a dark room in a minimal time." While it is assumed that the search range corresponds to a room filled with furniture, to find the cat, however, minimal lighting is needed.

Regarding architecture, optimization should be understood as rationalizing technical solutions by undertaking solutions that are the most beneficial at the early design stage. The more advanced the building process is, the more expensive the building optimization is. In a search for savings in contemporary architecture, an increasingly frequent phenomenon is a multivariate concept deepened by thorough analyzes carried out on several levels - these include structural analysis (structural optimization), energy, urban planning, materials. All these elements have an impact on the form - its technical and technological efficiency and artistic expression. Nevertheless, another development can be observed in the parameterization of elements involved in the form's design, which allows it to iterate the design process, leading to the "emergence" of practical solutions [6]. Regardless of the chosen technique, achieving the intended goal requires a well-thought-out sequence of actions and the use of the right tools at the right time. Optimal form-finding with the use of parametric tools requires "algorithmic thinking" [7].

\section{Background}

Contemporary architect's tools are computer programs used at all creative stages of work, from the early visions to detailed technical drawings. The digitization of the design process allows the integration of various technical disciplines. Thanks to integrating digital systems, it is possible to design in tight cooperation between multiple branches, interdependently searching for rational solutions, which often are a creative compromise for the target solutions [8]. The interdisciplinary design of gridshells leads to sophisticated structural systems that are often characterized by unobvious, complex shapes, with their geometries referring to free fractal structures, sometimes going beyond Euclidean geometry paradigms [9].

\subsection{Biomimetic inspirations}

In the search for the new architectural gridshell solutions, bionic patterns play an essential role. With the

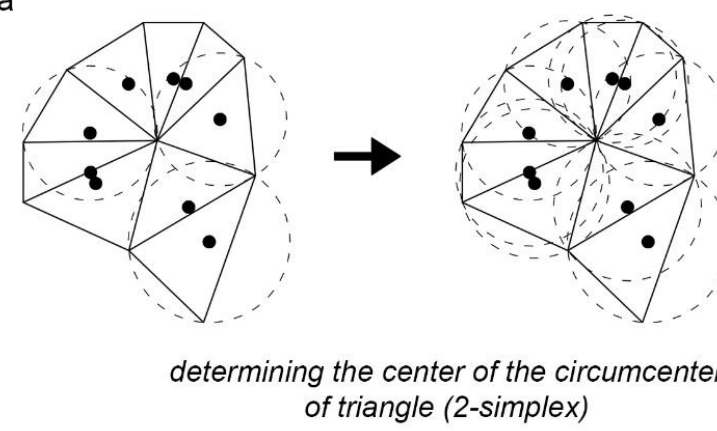

b

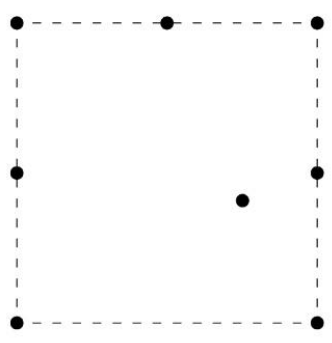

set of points

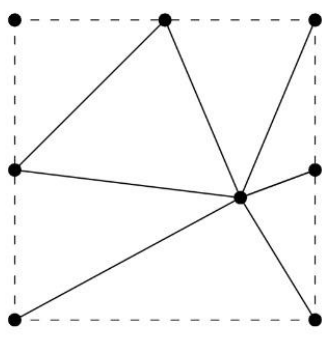

Delaunay triangulation for set of points

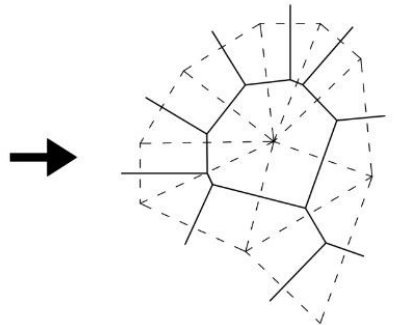

Voronoi diagram - dual graph

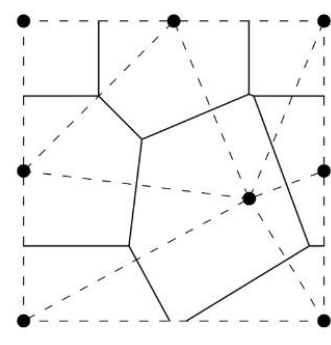

Voronoi Diagram for set of points

Fig. 1. Delaunay triangulation and Voronoi diagram - the divisions are also a dual graph with each other; $\boldsymbol{a}$ - creation of the Voronoi diagram based on a Delaunay's triangular division grid; $\boldsymbol{b}$ - Delaunay triangulation and Voronoi diagram for a given group of points; (author's compilation). 

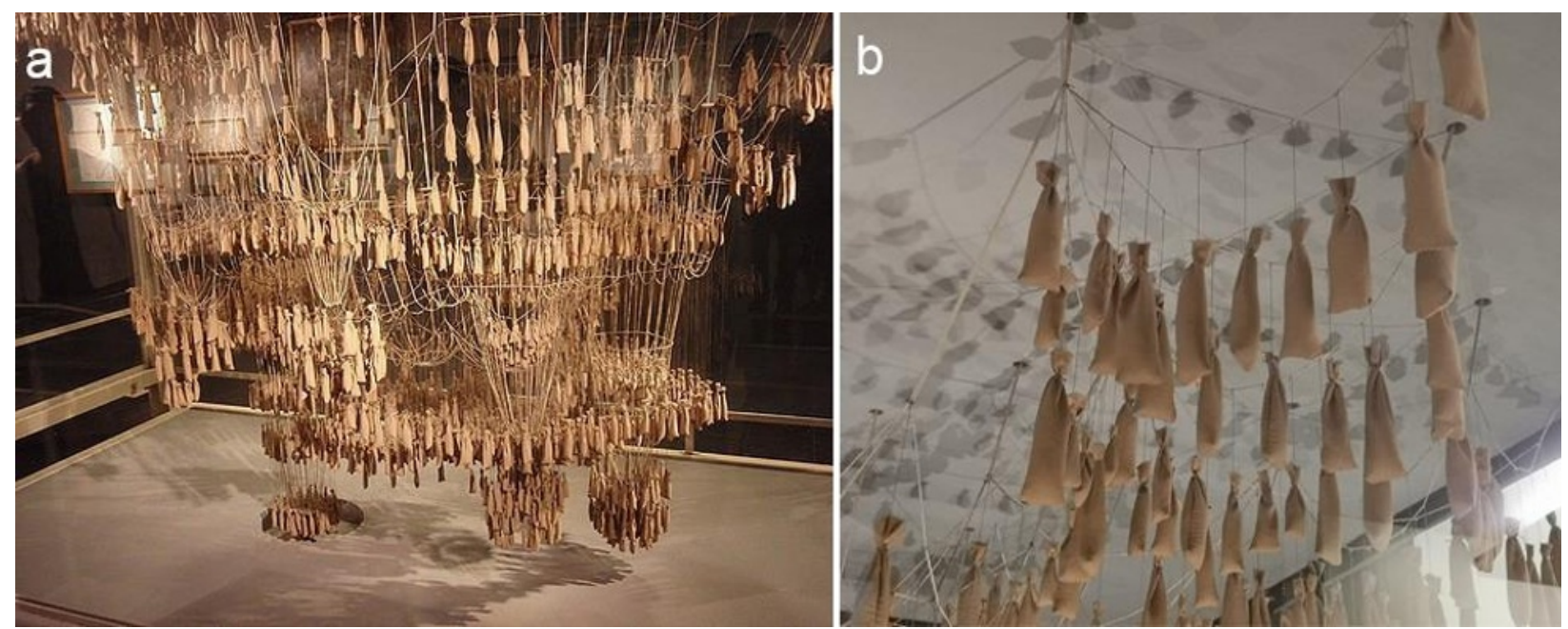

Fig. 2. A. Gaudi - model research; $\boldsymbol{a}$-1:10 model of Sagrada Familia; $\boldsymbol{b}$-detail of the binding of the weights.

development of research tools, designers learn about the formation of living organisms' surface structures. The knowledge becomes an inspiration in the design of loadbearing structures and broadly understood civil engineering and materials science.

One of the rules of mathematics and geometry inspired by bionics is the Delaunay triangulation (the characteristic triangular division of the surface, defined by the Russian mathematician Boris Delone in 1934), opposite to regular tessellation known and used in architecture [10]. Delaunay Triangulation $(\mathbf{T})$ denotes such a spatial division $\left(\mathbf{R}^{\mathbf{n}+1}\right)$ into convex polygons (simplexes) that two simplexes from $\mathbf{T}$ share a common wall or do not share a standard part; each limited set of $\mathbf{R}^{\mathbf{n}+1}$ shares its parts only with some finite $\mathbf{T}$ simplexes; the interior of a sphere circumscribed in any $\mathbf{T}$ simplex does not contain any simplex vertices of $\mathbf{T}$. The Delaunay Triangulation is also a dual graph of a Voronoi Diagram, shown in the figure below (Fig. 1) [11].

While structural optimization, the form needs to be determined along with the rational cross-sections of the elements related to the choice of appropriate materials. The rational design choices and following the systems of forces have the most significant influence on form aesthetics. In this context, the following issues deserve special attention: designing structural surfaces' form and discretization. One of the more significant exploration directions is topologies describing structural deformations, leading to rational solutions. The most famous example of the use of topology in architecture is the catenary curve described in the Renaissance (the first sketches of suspended chains can be found in the works of Leonardo da Vinci). The Simon Stevin manual from 1634 states that the rope hanging freely between supports is shaped parabolically. At the same time, Christian Huygens described the geometry of the catenary curve (Latin Catena - chain), proving that the curve, similar in its shape to a parabola, is an independent creation. In the last century, Antonio Gaudi, whose work on chain models lasted ten years, was the architect who contributed most to the research on catenary curves. Among others, the Sagrada Familia's physical model in a 1:10 scale was created at that time.

\subsection{Parametric tools}

One of the more critical issues in the optimal design of structural forms is the surface's discretization. The selection of the appropriate method of division and the indication of the mesh density have a fundamental impact on the structure's efficiency. An example of such optimization is the construction project by the engineers Bollinger + Grohmann made for Kunsthaus Graz. The structure was hidden, so the aesthetic features were not visible- the optimization consisted of minimizing steel consumption by choosing the right gridshell structure. For the curvilinear surface designed by the architects, a practical framework consisting of triangles and hexagons was designed - in place of the characteristic skylights. 


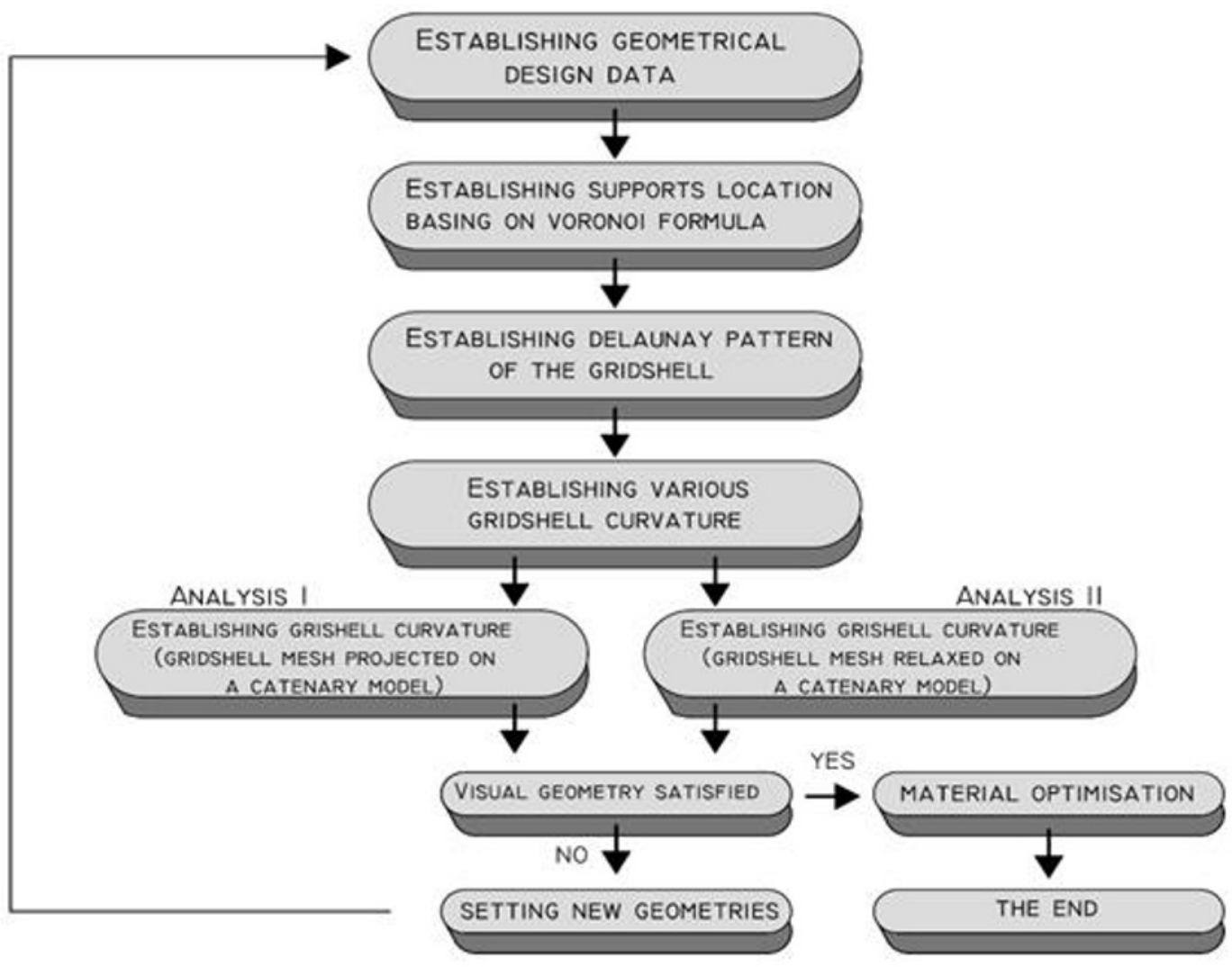

Fig. 3. Form-finding analysis flowchart (author's compilation).

Apart from the Finite Elements Method, which leads to effective structural solutions, many other methods are still used to discretize contemporary surfaces. One of them is Delaunay's Triangulation, which uses the Voronoi Diagram's bionic pattern - being its dual graph. An accessible tool for delineating Delaunay divisions is the Grasshopper plug-in for the Rhinoceros program, which allows the discretization of the surface based on the adopted geometric parameters. However, the modeled gridshell must then be subjected to structural analysis - this requires the cooperation between two different programs. The model is generated with a specified by the algorithm geometry, and intuitive assumptions are subjected to further optimizations. One of the optimization methods can be Dynamic Relaxation - an additional function of the Grasshopper plug-in. It is a numerical method of computational modeling, which aims to find such a geometry that all forces are in equilibrium [12]. It leads to a rationalization of solutions, including reducing the structure's total weight, which directly impacts reducing the amount of material used and reducing later construction costs [13] [14]. This method makes topological transformations of a given structure, leading to the relaxation of nodes.

\subsection{Structural optimization}

At the end of the twentieth century, structural forms' optimization has become a multidisciplinary issue - combining architecture and civil engineering and areas such as biology, chemistry, computer science, or mathematics. Multithreading manifesting itself among others about bionic patterns is visible in numerous designers such as A. Gaudi, F. Otto, S. de Chateau, who, without computer programs, created valuable, iconic objects. The digitization of the design environment visible at the turn of the 20th and 21 st centuries provided new tools that eased the design work (graphic presentation - 3D model, creation of technical documentation). The algorithmization has also provided the basis for the digital optimization of structural forms, enabling the reproduction of bionic structures and behaviors, thus indicating a new, morphic language of architecture [15] [16] [17]. The new generations of tools supporting the creation of architecture enforce the search for rational solutions. Bionic inspiration is made possible through the recreation of advanced geometry, mapping of systems and their behaviors in terms of minimal energy consumption, or changing the properties of materials at the nanoscale. The design of organic forms is also possible due to the development of construction techniques and manufacturing processes, including the improvement of building materials and components [18].

\section{Research methodology}

Referring to selected mathematical models and optimizing structural surfaces discussed in the previous 
a

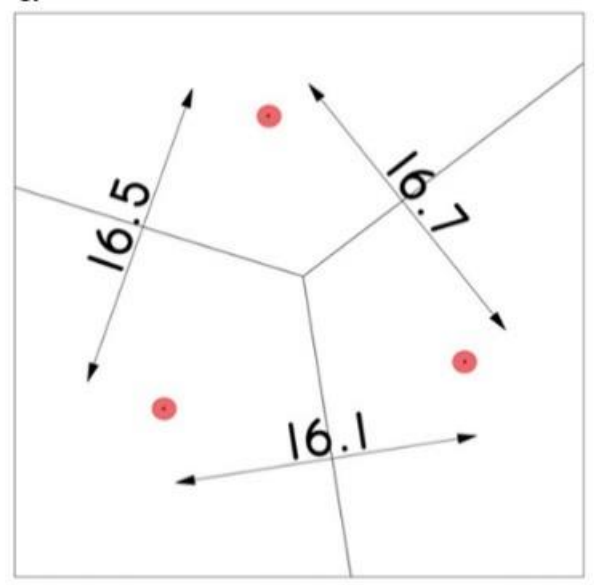

b

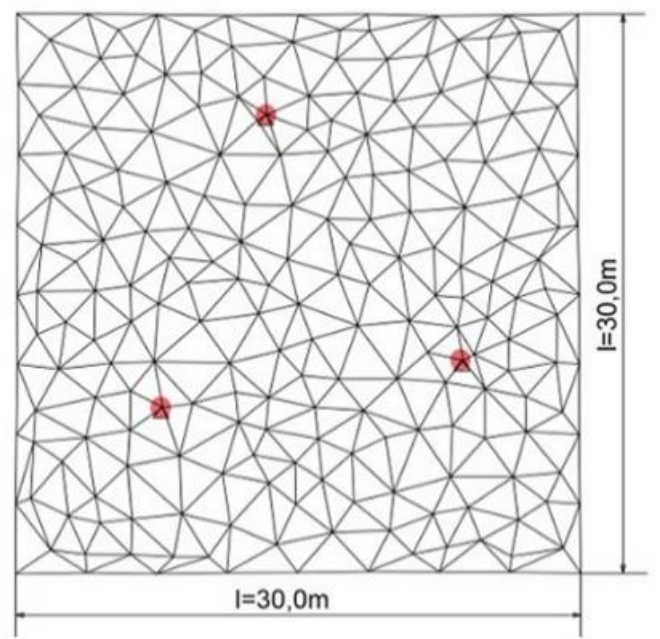

c

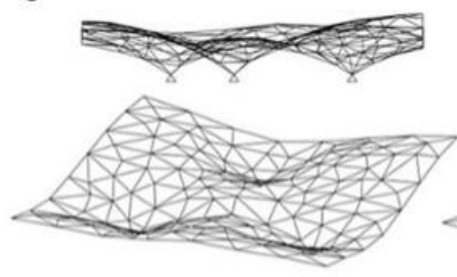

$1 / 5$

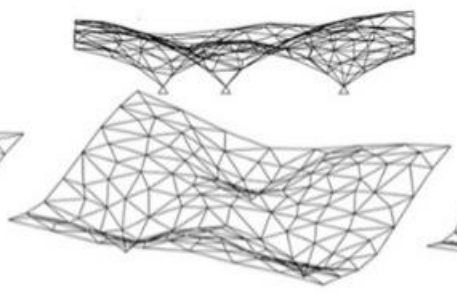

$1 / 4$

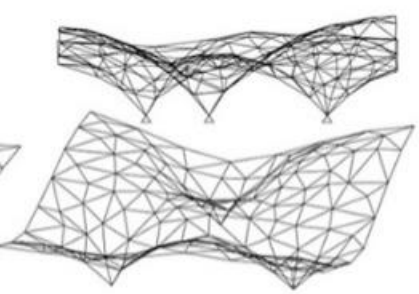

$1 / 3$

Fig. 4. Geometry assumptions: $\boldsymbol{a}$ - scheme of supports location and determining the Voronoi polygons of a comparable surface; $\boldsymbol{b}$-Diagram of Delaunay divisions with the maximum $4.5 \mathrm{~m}$ long beams; $\boldsymbol{c}$-curvature heights.

chapters, the relaxation of the nodes on the canopies structure was examined. The examples of structures created thanks to the Grasshopper plug-in for the Rhinoceros program and the Kangaroo2 plug-in for Grasshopper (this plug allows simulation of gravitational forces on the structure, thanks to which it is possible to create catenary models), then analyzed structurally using Robot Structural Analysis programs.

Access to computer-aided design tools significantly accelerated the search for the optimal structural form. An example of such a tool is the Grasshopper-Karagaroo2 plug-in used in the following tests, which assigned loads imitating physical forces to the pre-created mesh. The ability to change the acting forces, the extensibility of the elements, and the supports' placement generate a system of solutions subjected to structural analysis in the next phase. The study compares grids projected onto the created geometry(Analysis 1) with the grids generated utilizing Dynamic Relaxation (Analysis 2). The gridshell analysis results described below were built on the basic algorithm (see Fig. 3) and geometrical assumptions (see Fig. 4.).

The case study was conducted on free-formed pavilion canopies with the following assumptions:

-the canopy is on a square plan with a total area of $900.0 \mathrm{sq} \mathrm{m}$;

- the roofing is based on three supports, determined using the Voronoi formula. The supports are positioned so that they form an almost equilateral triangle, being at the same time the centers of a $300.0 \mathrm{sq} \mathrm{m}$ area;

-Delaunay triangulation was used to determine the gridshell structural divisions, with an assumption of each bar's maximum length not more than $4.5 \mathrm{~m}$;

-Analysis 1 was based on preset catenary curve geometry, on which the nodal points of the gridshell were projected (nodes moved only in Z-axis);

-Analysis 2 is based on nodal Dynamic Relaxation in mesh (nodes moved in Z-axis and adjusted to force flow also in the $\mathrm{X}$ - and Y-axis)(see Fig. 5.).

\section{Results and discussion}

\subsection{Analysis 1}

The Delaunay triangulation, initially applied to a flat geometry, was then projected onto the preset catenary geometry (node points were only moved in the Z-axis so that they were on the surface of the curvature) determined according to the catenary model principles:

- the deflection is determined by the height of the middle point to the average support span ratio. The following three options have been proposed depending on the height of the center point (Fig. 4c.):

-Variant A1 - height to the support span ratio: 1/5

-Variant B1 - height to the support span ratio: 1/4

-Variant C1 - height to the support span ratio: 1/3 


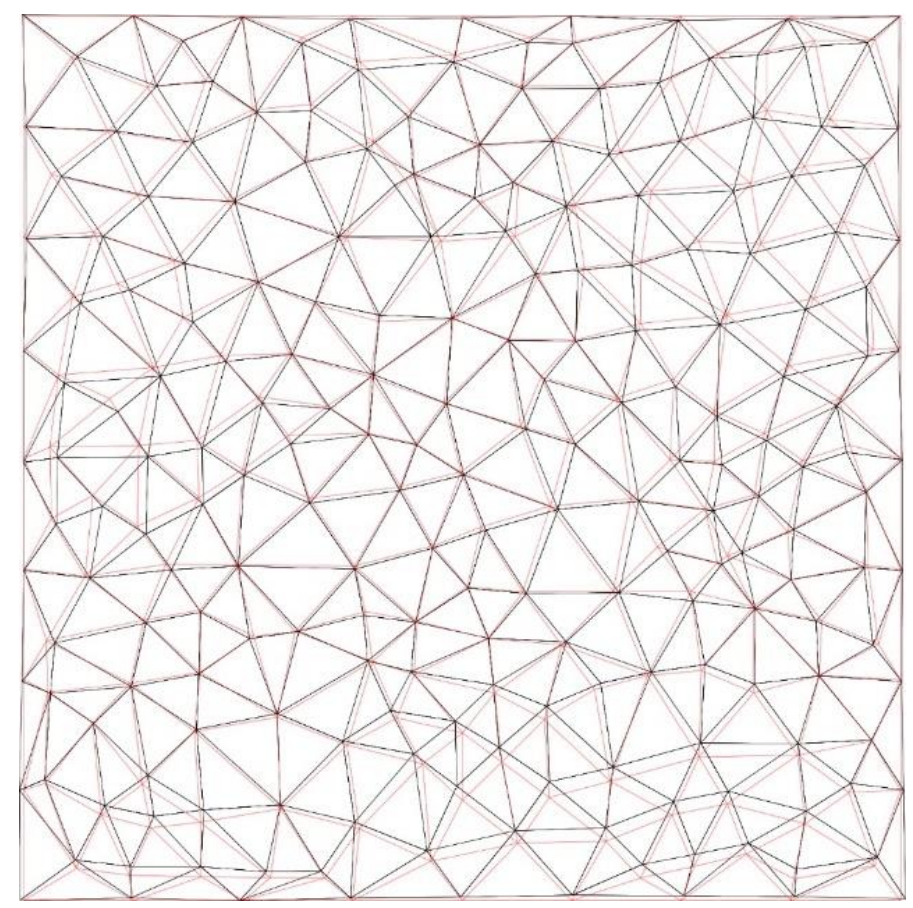

Fig. 5. Ilustration of overlapping variants $C$ from Analysis 1 and Analysis 2 (the $1 / 3$ ratio), showing the degree of changes in the geometry of Delaunay divisions (red) due to topological transformations using the logic of chain models (black).

Uniform closed THEX (hexagonal tubes), TRON (round tubes), and TREC (rectangular tubes) profiles made of S355 steel were adopted for all the layouts. One of the assumptions was the homogeneity of the structure; hence, one of the most effective external dimensions of the profile was selected (although individual beams in the structure do not work equally). Simultaneously, it was possible to change the wall thickness of a given profile - though, from the outside, the profiles should look the same. Due to all variants' same starting geometry, a maximum deformation value of $19.5 \mathrm{~cm}$ was assumed. In the search for effectiveness, the minimum mass criterion for the entire roof was adopted. Due to the variable roof area, the weight per $1.0 \mathrm{~m}^{2}$ of the canopy was also considered an important parameter.

Two profiles differing in wall thickness were selected for all variants. Based on the adopted weight criterion, the most effective variant was Variant C1 (total weight of $73253 \mathrm{~kg}$ ). Although the roofing's largest area characterized this variant, it was characterized by the smallest weight per $1.0 \mathrm{~m}^{2}$ of the roof, which amounted to $72.38 \mathrm{~kg} / \mathrm{m}^{2}$ - about $30 \%$ less than the most unfavorable option. Additionally, in all the analyzed variants, the TRON (round) steel profile was the most effective(see Tabel 1).

\subsection{Analysis 2}

Analysis 2 adopts the same Delaunay Triangulation pattern for the gridshell form. Nodal points have been released in the $\mathrm{X}$ - and $\mathrm{Y}$-axis to enable their Dynamic Relaxation, following the acting forces. 
Table 2. Results of chain structure analysis using dynamic relaxation with a maximum beam length of $4.5 \mathrm{~m}$.

\begin{tabular}{|c|c|c|c|c|c|c|c|c|c|c|}
\hline \multirow{2}{*}{ 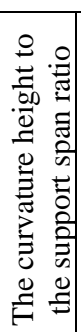 } & \multirow{2}{*}{ 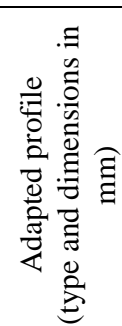 } & \multirow{2}{*}{ 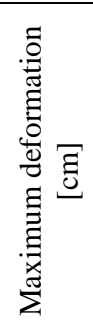 } & \multicolumn{2}{|c|}{ 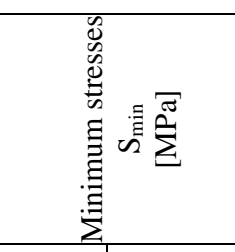 } & \multicolumn{2}{|c|}{ 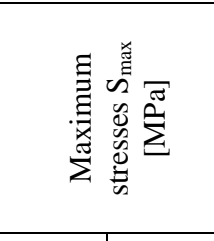 } & \multirow{2}{*}{ 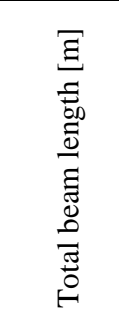 } & \multirow{2}{*}{ 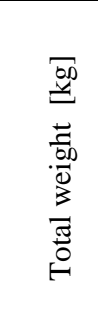 } & \multirow[t]{2}{*}{ 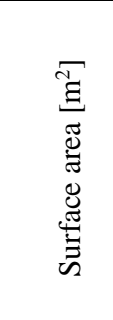 } & \multirow{2}{*}{ 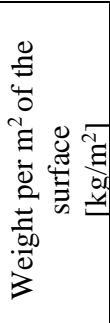 } \\
\hline & & & $\stackrel{\text { }}{\stackrel{\Xi}{\Xi}}$ & ·灵 & 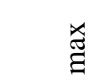 & . & & & & \\
\hline $\begin{array}{l}\text { A2. } \\
1 / 5\end{array}$ & $\begin{array}{c}\text { TRON } \\
355 \times 8 \\
355 \times 6,3\end{array}$ & 16,1 & 93,54 & $-269,98$ & $\begin{array}{c}233,5 \\
3\end{array}$ & $-89,85$ & 1531,06 & 83947 & 945,73 & 88,76 \\
\hline $\begin{array}{l}\mathrm{B} 2 . \\
1 / 4\end{array}$ & $\begin{array}{c}\text { TRON } \\
323 \times 6,3 \\
323 \times 5,6\end{array}$ & 16,4 & $\begin{array}{c}118,3 \\
6\end{array}$ & $-311,90$ & $\begin{array}{c}230,4 \\
3\end{array}$ & $\begin{array}{c}- \\
101,7 \\
4\end{array}$ & 1548,05 & 68413 & 968,64 & 70,68 \\
\hline $\begin{array}{l}\mathrm{C} 2 . \\
1 / 3\end{array}$ & $\begin{array}{c}\text { TRON } \\
273 \times 6,3 \\
273 \times 5,0\end{array}$ & 16,6 & $\begin{array}{c}131,0 \\
8\end{array}$ & $-337,01$ & $\begin{array}{c}234,9 \\
0\end{array}$ & $-96,66$ & 1581,37 & 54094 & 1011,95 & 53,46 \\
\hline
\end{tabular}

Thanks to the generative algorithm, the adopted variants change the geometrical layout to a small degree - changes are unnoticeable in the scale of the entire gridshell. As the constructional analysis shows, the change in the structural layout has significantly improved the results. Analysis 2 adopts variants analogous to Analysis 1 with the same center point height to the support span ratio:

-Variant A2 - height to the support span ratio: 1/5

-Variant B2 - height to the support span ratio: 1/4

-Variant C2 - height to the support span ratio: 1/3

Similarly to Analysis 1, homogeneous closed profiles were adopted by selecting from the THEX (hexagonal tubes), TRON (round tubes), and TREC (rectangular tubes) profiles made of S355 class steel. For all the variants, the round tube also proved to be the most effective. Variant C2 (with the $1 / 3$ ratio) reached the smallest total mass (analogously as in Analysis 1) equal to $54094 \mathrm{~kg}$. The weight ratio per $1.0 \mathrm{~m}^{2}$ of the canopy was $53.46 \mathrm{~kg} / \mathrm{m}^{2}$ (see Tabel 2 ).

\subsection{Discussion}

In Analysis 2, there is a significant improvement in the efficiency of structures with the layouts in Analysis 1. There is also a visible relationship between Analysis 1 and Analysis 2, where all the results of A2, B2, C2 are much more favorable(see Fig. 6.).

Variant A: weight improvement: the A2 variant is $13,390 \mathrm{~kg}$ lighter than the A1 variant, which results in $13.76 \%$ lower material consumption;

Variant B: weight improvement: the B2 variant is

Table 1. Results of analyzes of structures with a maximum projected length of beams of $4.5 \mathrm{~m}$.

\begin{tabular}{|c|c|c|c|c|c|c|c|c|c|c|}
\hline \multirow{2}{*}{ 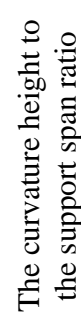 } & \multirow{2}{*}{ 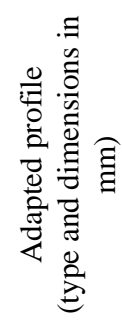 } & \multirow{2}{*}{ 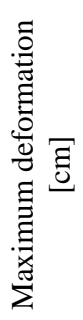 } & \multicolumn{2}{|c|}{ 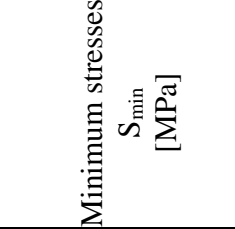 } & \multicolumn{2}{|c|}{ 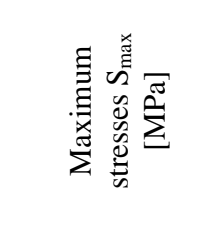 } & \multirow{2}{*}{ 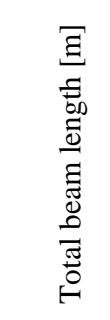 } & \multirow{2}{*}{ 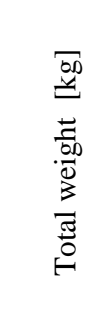 } & \multirow{2}{*}{ 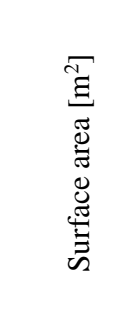 } & \multirow{2}{*}{ 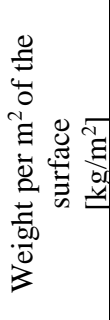 } \\
\hline & & & 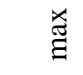 &.$\Xi$ & 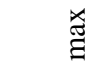 & 寻 & & & & \\
\hline $\begin{array}{l}\text { A1. } \\
1 / 5\end{array}$ & $\begin{array}{c}\text { TRON } \\
406 \times 8 \\
406 \times 6,3\end{array}$ & 18,4 & 83,92 & $-221,56$ & $\begin{array}{c}322,7 \\
2\end{array}$ & $-80,17$ & 1536,09 & 97337 & 943,75 & $\begin{array}{c}103,1 \\
3\end{array}$ \\
\hline $\begin{array}{l}\text { B1. } \\
1 / 4\end{array}$ & $\begin{array}{c}\text { TRON } \\
355 \times 6,3 \\
355 \times 8\end{array}$ & 19 & $\begin{array}{c}105,1 \\
1\end{array}$ & $-225,24$ & $\begin{array}{c}325,9 \\
6\end{array}$ & $-82,8$ & 1558,42 & 92454 & 965,17 & 95,79 \\
\hline $\begin{array}{l}\text { C1. } \\
1 / 3\end{array}$ & $\begin{array}{c}\text { TRON } \\
323 \times 5,6 \\
323 \times 6,3\end{array}$ & 19,4 & 118 & $-203,83$ & $\begin{array}{c}252,4 \\
6\end{array}$ & $-77,35$ & 1593,86 & 73253 & 1012,01 & 72,38 \\
\hline
\end{tabular}


$24,041 \mathrm{~kg}$ lighter than variant $\mathrm{B} 1$, which results in 26.00\% lower material consumption;

Variant C: weight improvement: the $\mathrm{C} 2$ variant is 19 $159 \mathrm{~kg}$ lighter than variant $\mathrm{C} 1$, which results in $26.15 \%$ lower material consumption;

It should also be noted that the $\mathrm{C}$ variants in each of the Analyzes, despite the most extensive length of beams, achieve the smallest total weight.

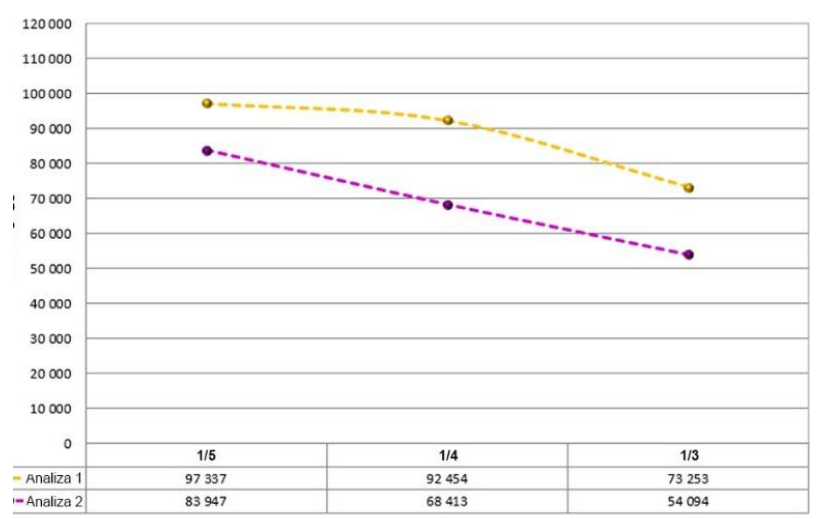

Fig. 6. List of results obtained from the analysis of individual variants from Analysis 1 and Analysis 2.

Topological optimization of the structural surfaces, using catenary models' algorithms, allowed to reduce structural material consumption on average by $21.97 \%$.

\section{Conclusion}

The current development of digital technologies enables the shaping and realization of forms with complex geometry. However, regardless of the tools available, the critical element is invariably the awareness of the creation process and determining these activities' purposes [19]. The integration of elements of art and technology in parametric designing is necessary for the era of modern trends and generative tools of the architect's work, in particular as the number of fields that take part in the process of creating architecture (biology, information technology, programming) is continuously developing [20]. Bionics inspiration in contemporary architecture and construction is an attractive and complex phenomenon. The aesthetic side of structural form shaping remains for subjective evaluation. In shaping the spatial form through morphological analysis, it is possible to find an increased share of logic and cool calculation in creating an artwork - assuming that what is right (functional, rational) is also beautiful.

\subsection{Limitations and future scope of the study}

The limitation of such research is insufficient knowledge in associating IT tools such as generative and parametric designing into interdisciplinary structural optimization. The search for logical solutions leads to bionic systems whose beauty results from Nature. The search for minimal consumption is the main aim of architects and structural designers. Nevertheless, mathematical models inspired by living organisms' morphology can rationalize technical solutions when it is justified and implied according to the construction logic. An example of such action is the analysis carried out in the above study - where the use of a chain model was dictated by the model of physical forces acting on the assumed structure. The conducted analyses also confirmed the assumption that the form's shaping has the most significant influence on structural efficiency [21]. Therefore the pursuit of shape optimization should be the initial parameter at the early stage of creating an architectural concept.

\section{References}

1. M. S. Aziz i A. El Sherif, „Biomimicry as an approach for bio-inspired structure witht he aid of computation," Alexandria Engineerign Journal, (2015)

2. J. N. Richardson, S. Adriaenssens, R. F. Coelho and P. Bouillard, "Coupled form-findind and grid optimisation approach for single layer grid shells," Engineering Structures, 53, 230-239 (2013).

3. M. Pawlyn, Biomimicry in architecture (London: Riba Publisher, 2016).

4. K. Januszkiewicz, "Projektowanie parametryczne oraz parametryczne narzędzia cyfrowe w projektowaniu architektonicznym (eng. Parametric design and parametric digital tools in architectural design)," Architecturae et Artibus, 3 (2016).

5. N. A. Buzalo, S. O. Versilov, I. D. Platonova i N. G. Tsaritova, „Energy efficient building structures based on grodshell," w IOP Conf. Series: Materials Science and Engineering, 2018.

6. S. H. Dyvik, M. Luczkowski, J. H. Mork, A. Ronnquist i B. Manum, „Design of freeform gridshell structures- Simplifying the parametric workflow," w AIBSE Symposium 2019 Guimaraes: Towards a Resilient Built Environment- Risk and Asset Management, Guimaraes, 2019.

7. W. J. Lewis, "Computational form-finding methods for fabric structures," in Proceedings of the Institution of Civil Engineers-Engineering and Computational Mechanics (2008).

8. R. Maia Avelino, O. Bavarel and A. Lebee, Design strategies for gridshells with singularities, Journal of the international association for Shell and Spatial Structures (2019).

9. E. Gawell, Synergia formy przestrzennej i statyki $w$ optymalnym ksztaltowaniu struktur prętowych (eng. Synergy of spatial form and statics in the optimal shaping of bar structures), Warsaw: Ph.D. Dissertation, dept Arch. WUT (2015).

10. R. Dyer, H. Zhang and T. Moller, A survey of Delaunay structures for surfacerepresentation, GrUVi Lab, School of Computing Science, Simon Fraser University, 1601 2009. [Online]. Available: https://www.researchgate.net/publication/22878383 
6_A_survey_of_Delaunay_structures_for_surface_r epresentation. [Accessed 1311 2020].

11. H. Hua, L. Hovestadt i P. Tang, Optimization and prefabrication of timber Voronoi shell, Structural and Miltidisciplinary Optimization (2020).

12. D. Veenendaal and P. Block, An overview and comparison of structural form finding methods, International Journal of Solids and Structures, 49, 3741-3753 (2013)

13. F. Tayeb, J. F. Caron, O. Baverel and L. Du Peloux, Stability and robustness of a $300 \mathrm{~m} 2$ composite gridhell structure, Construction and Building Materials (2013).

14. B. MR, Applications of dynamix relaxation to the topological design and analysis of cable, membrane and pneumatic structures, w Secon International conference on space structures (1975).

15. V. Alic and K. Persson, Form finding with dynamic relaxation and isogeometric membrane elements, Computer methods in applied mechanics and engineering, 300, $734-747$ (2016).

16. R. Mesnil, C. Douthe and O. Baverel, Non-standard patterns for gridshell structures: fabrication and srtuctural optimization, Journal of the International Association for Shell and Spatial Structures (2017).

17. A. Menges, Biomimetic design processes in architecture:morphogenetic and evolutionary computational design, Bioinspiration \&Biomimetics (2012)

18. A. Menges, Glynn, R. Glynn and M. Skvara, Fabricate. Rethinking design and construction, Riverside Architectural Press (2017)

19. S. Dixit, S. N. Mandal, J. V. Thanikal i K. Saurabh, Evolution of studies in construction prductivity: A systematic literature review (2006-2017), Ain Shams Engineering Journal (2019)

20. A. Stefańska, Reticulated Roof Structures Optimisation Based of Triangular, w Proceedings of the Creative Construction e-Conference (2020).

21. L. Wang, P. Janssen and G. Ji, SSIEA: a hybrid evolutionary algorithm for supporting conceptual architectural design, Artificial Intelligence for Engineering Design and Manufacturing, 1-19 (2020). 